Quim. Nova, Vol. 33, No. 4, 938-944, 2010

\title{
PROPRIEDADES DINÂMICAS DE FLUIDOS POR SIMULAÇÃO COMPUTACIONAL: MÉTODOS HÍBRIDOS ATOMÍSTICO-CONTÍNUO
}

\author{
Luciano T. Costa* \\ Departamento de Ciências Exatas, Universidade Federal de Alfenas, 37130-000 Alfenas -MG, Brasil \\ Mauro C. C. Ribeiro \\ Instituto de Química, Universidade de São Paulo, CP 26077, 05513-970 São Paulo - SP, Brasil
}

Recebido em 9/8/09; aceito em 13/11/09; publicado na web em 10/3/10

\begin{abstract}
COMPUTER SIMULATIONS OF DYNAMICAL PROPERTIES OF FLUIDS: ATOMISTIC-CONTINUUM HYBRID METHODS.
Computational methods for the calculation of dynamical properties of fluids might consider the system as a continuum or as an assembly of molecules. Molecular dynamics (MD) simulation includes molecular resolution, whereas computational fluid dynamics (CFD) considers the fluid as a continuum. This work provides a review of hybrid methods MD/CFD recently proposed in the literature. Theoretical foundations, basic approaches of computational methods, and dynamical properties typically calculated by MD and CFD are first presented in order to appreciate the similarities and differences between these two methods. Then, methods for coupling MD and CFD, and applications of hybrid simulations MD/CFD, are presented.
\end{abstract}

Keywords: molecular dynamics; computational fluid dynamics; hybrid methods.

\section{INTRODUÇÃO}

A dicotomia na visão da matéria como um meio contínuo ${ }^{1}$ ou composta por átomos ${ }^{2}$ acompanha a história do ocidente desde a Filosofia grega clássica. Na Física e Química modernas, essas visões são conciliadas uma vez que a descrição de contínuo considera médias locais de propriedades microscópicas sobre uma região grande do ponto de vista atômico. A descrição atomística envolve a evolução dinâmica de um grande número de variáveis, isto é, coordenadas e momento de cada partícula no caso da Mecânica Clássica, ou a função de onda do sistema no caso da Mecânica Quântica. A descrição contínua da matéria envolve um número pequeno de propriedades conservadas, isto é, massa, momento e energia, cuja evolução dinâmica é dada pelas equações de movimento apropriadas, as equações de Navier-Stokes da hidrodinâmica. ${ }^{3}$ Atualmente, metodologias bem estabelecidas para ambas estão disponíveis em procedimentos numéricos de simulação computacional de fases condensadas. Simulações clássicas de sistemas moleculares são implementadas em métodos de Monte Carlo (MC) ou de Dinâmica Molecular (MD), ${ }^{4}$ enquanto que simulações de meios contínuos são implementadas em métodos de dinâmica de fluidos computacional (CFD). ${ }^{5}$

Coeficientes de transporte, tais como viscosidade e condutividade térmica, necessários às equações de Navier-Stokes em CFD, podem ser calculados por simulações MD. Métodos de simulação MD de equilíbrio são mais comuns, a partir dos quais coeficientes de transporte são obtidos pelas relações de Green-Kubo, ou seja, como a integral da função de correlação no tempo de flutuações da propriedade relacionada a um determinado coeficiente de transporte. ${ }^{6,7}$ Alternativamente, métodos de simulação MD de não equilíbrio (NEMD) implementam uma estratégia análoga ao realizado experimentalmente, uma vez que o sistema é submetido a uma perturbação externa e a resposta é mensurada. ${ }^{4,8}$ Evidentemente, simulações MD são necessárias quando se deseja a interpretação microscópica de um dado coeficiente de transporte, mas são computacionalmente proibitivas quando se deseja uma descrição do sistema em escala macroscópica, para a qual métodos CFD são necessários.

\footnotetext{
*e-mail: costalt@gmail.com
}

Assim como a técnica CFD ao longo dos anos adquiriu confiabilidade por parte da indústria, cada vez mais podemos constatar o uso da técnica MD em pesquisas de aplicação industrial e sua consolidação como ferramenta na predição de novos materiais. Potenciais aplicações de simulações MD nas mais diversas áreas da ciência têm despertado interesse de parcerias acadêmico-empresariais com o objetivo de estudar o comportamento de fluidos em condições de difícil reprodução experimental ou como suporte ao dado experimental.

Neste trabalho, apresentam-se as principais rotas de cálculo de propriedades dinâmicas de fluidos, enfatizando o método híbrido contínuo-atomístico como técnica promissora no estudo do comportamento de fluidos sob diferentes condições de escoamento. Neste sentido, é necessário apresentarmos alguns aspectos teóricos e computacionais fundamentais das simulações de contínuo e atomísticas separadamente.

\section{FUNDAMENTOS TEÓRICOS}

\section{Equações de Navier-Stokes}

O escoamento de um fluido pode ser laminar ou turbulento, sendo que neste trabalho estamos particularmente interessados no primeiro, onde camadas finas deslizam umas em relação às outras. O escoamento de fluidos compressíveis e incompressíveis, com fluxo turbulento ou laminar, pode ser modelado pelas equações de Navier-Stokes formuladas no século XIX por C. Navier (1822) e G. Stokes (1845)., 3 O fluido é considerado, sob o ponto de vista macroscópico, como um contínuo, onde a estrutura discreta da matéria e os movimentos das moléculas podem ser desprezados. Um elemento de fluido contém um número suficientemente grande de moléculas de tal modo que não nos ocupamos com as moléculas individuais, ou seja, grandezas macroscópicas representam médias sobre todas as moléculas que compõem um elemento de fluido.

Três princípios de conservação governam a formulação de Navier-Stokes: conservação de massa, momento e energia. Com o propósito de ilustrar o tipo de equações resolvidas numericamente em CFD, consideremos apenas a equação resultante da conservação da massa para um escoamento bidimensional na direção $x$ tal como na Figura 1, onde a componente do vetor velocidade $\mathbf{v}$ ao longo de $x$ é definida por $u$. Em um intervalo de tempo pequeno, $\Delta t$, o fluido 
percorre a distância $l=u \Delta t$. Se multiplicarmos essa distância pela área $S$, obteremos o volume do fluido, o qual, quando multiplicado pela densidade $\rho$, fornece a massa total de fluido entre as placas: $:^{3,5}$

$m=u \Delta t S \rho$

A vazão de massa, isto é, descarga, é a quantidade de massa que atravessa a área $S$ por unidade de tempo. A variação no tempo da densidade do fluido e a variação da massa por unidade de volume resultam na equação da continuidade, a qual, no caso particular da Figura 1, é:

$$
\frac{\partial \rho}{\partial t}+\frac{\partial(\rho u)}{\partial x}=0
$$

A forma geral desta equação é:

$$
\frac{\partial \rho}{\partial t}+\nabla \cdot(\rho \mathbf{v})=0
$$

onde operador nabla é $\nabla=\hat{x} \frac{\partial}{\partial x}+\hat{y} \frac{\partial}{\partial y}+\hat{z} \frac{\partial}{\partial z}$, sendo $\hat{\imath}$ um vetor
unitário na direção $i$.

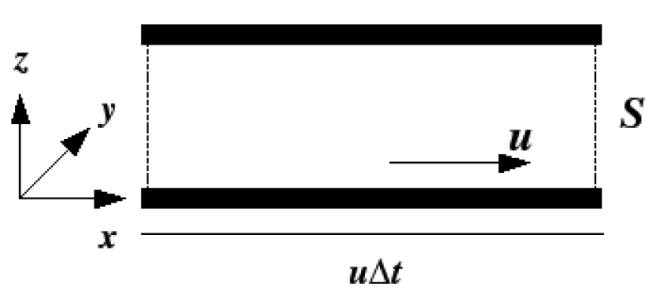

Figura 1. Modelo de escoamento bidimensional de um fluido entre duas paredes fixas, com área da seção transversal S e u a componente de velocidade ao longo da direção $x$

Além do fluxo de massa, pode haver fluxo de momento e energia com as equações de conservação correspondentes, as quais, em conjunto com a equação da continuidade, são conhecidas como equações de Navier-Stokes. ${ }^{3,5}$

\section{Mecânica estatística}

Consideremos um sistema contendo átomos ou moléculas em um estado definido pelo número de partículas $N$, volume $V$ e temperatura $T$ constantes. Podemos imaginar este sistema como uma amostra de um elemento muito pequeno de volume de fluido do modelo da Figura 1. Um conjunto de cópias desse sistema define o ensemble canônico $(N, V, T)$. A Mecânica estatística calcula probabilidade de uma determinada propriedade macroscópica no espaço de fase do sistema de partículas. O espaço de fase do sistema é dado pelas coordenadas $\mathrm{r}$ e momento $\mathrm{p}$ das partículas, portanto, no caso de um sistema com $N$ partículas, é um espaço de $6 N$ dimensões ( $3 N$ coordenadas e $3 \mathrm{~N}$ momento). No ensemble canônico, a probabilidade de encontrar um sistema com energia interna $E_{i}$ é dada pela distribuição de Boltzmann:4,6-9

$$
P_{i}=\frac{e^{-\beta E_{i}}}{\sum_{i} e^{-\beta E_{i}}}
$$

onde $\beta=\frac{1}{k T}, T$ é a temperatura e $k$ a constante de Boltzmann. O

valor esperado de uma propriedade qualquer $A(\mathrm{r}, \mathrm{p})$ é a média sobre as cópias do sistema:

$$
\langle A\rangle=\sum_{i} P_{i} A_{i}=\frac{\sum_{i} e^{-\beta E_{i}} A_{i}}{\sum_{i} e^{-\beta E_{i}}}
$$

No caso de um problema clássico, esta relação é dada pela forma integral:

$\langle A\rangle=\frac{\iint d \mathbf{p} d \mathbf{r} e^{-\beta E} A(\mathbf{r}, \mathbf{p})}{\iint d \mathbf{p} d \mathbf{r} e^{-\beta E}}$

Esta média sobre o ensemble representa a média sobre todas as configurações microscópicas compatíveis com o estado termodinâmico considerado. A simulação pelo método de Monte Carlo baseia-se em uma amostragem estatística suficientemente grande de configurações microscópicas, mas este método não permite a investigação da dinâmica do sistema. Ao contrário, o método de Dinâmica Molecular (MD) fornece valores instantâneos $A(t)=A[\mathrm{r}(t), \mathrm{p}(t)]$, onde a evolução da propriedade é acompanhada ao longo de um tempo de observação, $t_{o b s}$, e é razoável aproximar a média sobre o ensemble pela média sobre as configurações geradas pela simulação. Na prática, a média no tempo é dada por: ${ }^{4}$

$$
\langle A\rangle_{\text {tobs }}=\frac{1}{t_{\text {obs }}} \sum_{t=1}^{t_{\text {obs }}} A(t)
$$

Portanto, a Mecânica estatística é o fundamento teórico para o cálculo de propriedades dinâmicas por simulações MD.

\section{Métodos computacionais}

\section{Dinâmica de fluidos computacional}

A dinâmica de fluidos computacional, CFD, utiliza métodos numéricos para simulação de fenômenos que envolvem o fluxo de fluidos com ou sem troca de calor. A solução numérica das equações de Navier-Stokes permite obter distribuições de velocidade, pressão e temperatura na região de escoamento. $\mathrm{O}$ total de variáveis desconhecidas é maior que o número de equações, de tal modo que a solução é possível com equações adicionais baseadas na equação de estado do sistema, $P=P(\rho, T)$, sendo $P$ a pressão. Simplificações podem ser postuladas dependendo do tamanho e geometria do sistema e condições de contorno podem ser introduzidas para a solução numérica das equações, por exemplo, velocidade relativa zero entre uma superfície sólida e a camada adjacente de fluido. ${ }^{5,10-12}$

Evidentemente, diversos métodos numéricos para a solução das equações de Navier-Stokes estão disponíveis na literatura. Ilustraremos o procedimento com os métodos de diferenças finitas, os quais são particularmente interessantes pela eficiência computacional e por utilizarem a forma diferencial das leis de conservação. ${ }^{5,10-12}$ Considere um corte transversal na região contínua de fluido da Figura 1, conforme mostrado na Figura 2, na qual uma malha de pontos é desenhada, onde $\Delta x$ e $\Delta y$ representam diferenças finitas, igualmente espaçadas ou não, nas direções $x$ e $y$. Se $u_{i j}$ é a componente $x$ da velocidade no ponto $P$, então $u_{i+1, j}$ pode ser expandido em série de Taylor em torno de $(i, j)$ e os valores obtidos numericamente para toda a malha. Assim, o contínuo é tornado discreto e a derivada de uma função qualquer $f(x)$ é aproximada como diferença finita, tal como

$$
\left.\frac{\partial f}{\partial x}\right|_{i} \approx \frac{f_{i+1}-f_{i-1}}{2 \Delta x}
$$

No caso de equações envolvendo propriedades que além de discretizadas espacialmente também evoluem no tempo, por exemplo, 


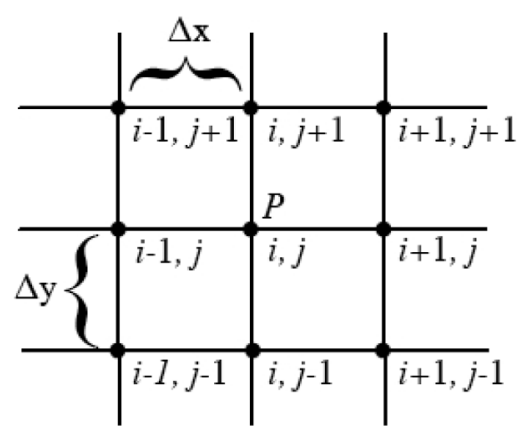

Figura 2. Representação de uma malha de pontos bidimensional para discretização espacial em simulações $C F D$

Equação 3, valores em tempo posteriores podem ser obtidos pelo chamado método de Euler: ${ }^{5}$

$$
A_{i}^{t+\Delta t}=A_{i}^{t}+\Delta t F\left(\alpha, A^{t}, \Delta x\right)
$$

onde a função $F\left(\alpha, A^{t}, \Delta x\right)$ depende da derivada espacial do problema em questão como ilustrado na Equação 8 e de eventuais parâmetros físicos $\alpha$, tais como coeficiente de difusividade térmica, viscosidade etc. Este método ilustra a obtenção da distribuição da propriedade $A$ partindo de valores iniciais e atualização ao longo do tempo.

\section{Dinâmica molecular}

A simulação MD clássica é baseada na evolução temporal de coordenadas e momento das $N$ partículas do sistema, $\left[\mathbf{r}^{N}, \mathbf{p}^{N}\right]$, onde $\mathbf{r}^{N}=\left(\mathbf{r}_{1}, \mathbf{r}_{2,}, \mathbf{r}_{3, \ldots}, \mathbf{r}_{N}\right)$ e $\mathbf{p}^{N}=\left(\mathbf{p}_{1}, \mathbf{p}_{2}, \mathbf{p}_{3, \ldots} \mathbf{p}_{N}\right)$. Simulação MD consiste na solução numérica das equações clássicas de movimento: ${ }^{4,9,13}$

$\mathbf{F}_{i}=m_{i} \frac{d^{2} \mathbf{r}_{i}}{d t^{2}}$

onde a força resultante na partícula $i$ é $\mathbf{F}_{i}=-\frac{\partial V}{\partial \mathbf{r}_{i}}$, sendo $V(r)$ é a função energia potencial. A partir de um microestado inicial, $\left[\mathbf{r}^{N}(0)\right.$, $\left.\mathbf{p}^{N}(0)\right]$, a integração das equações de movimento permite obter microestados em instantes sucessivos dada a função $V(r)$ assumida para o sistema. A função energia potencial é o cerne do método de simulação MD, incluindo termos intermoleculares e contribuições intramoleculares de estiramento de ligação, $R$, deformação de ângulo, $\theta$, e torção de ângulo diedro, $\phi:^{4,9,13}$

$V_{\text {total }}=\sum_{i j}\left\{4 \varepsilon_{i j}\left[\left(\frac{\sigma_{i j}}{r_{i j}}\right)^{12}-\left(\frac{\sigma_{i j}}{r_{i j}}\right)^{6}\right]+\frac{q_{i} q_{j}}{r_{i j}}\right\}+\sum_{\text {ligacão }} k_{R}\left(R-R_{e q}\right)^{2}$

$+\sum_{\text {angulo }} k_{\theta}\left(\theta-\theta_{\text {eq }}\right)^{2}+\sum_{\text {diedro }} k_{\phi}[1+\cos (n \phi-\delta)]$

onde $r_{i j}$ é a distância entre átomos $i$ e $j$ com cargas parciais $q_{i} \mathrm{e}$ $q_{j}$, $\varepsilon$ e $\sigma$ são os parâmetros de energia e distância do potencial Lennard-Jonnes. Esses são termos intermoleculares de curto alcance (Lennard-Jones) e interações de Coulomb de longo alcance. Os termos intramoleculares incluem parâmetros $R_{e q}$ e $\theta_{e q}$ para o comprimento e o ângulo de ligação de equilíbrio, respectivamente, as constantes de força $k_{R}, k_{\theta}$ e $k_{\phi}$ do potencial intramolecular de ligação, ângulo e diedro, respectivamente, e os parâmetros $n$ e $\delta$ determinam número de mínimos e fase do potencial de ângulo diedro. Os parâmetros do potencial são previamente ajustados pela reprodução de dados experimentais termodinâmicos, estruturais e dinâmicos, ou pela reprodução de dados obtidos por cálculos de Química Quântica para uma molécula isolada ou aglomerados de moléculas.

$\mathrm{O}$ algoritmo de velocity Verlet ilustra a integração numérica das equações de movimento e a evolução de $\left[\mathbf{r}^{N}(t), \mathbf{p}^{N}(t)\right]$ para $\left[\mathbf{r}^{N}(t+\Delta t)\right.$, $\left.\mathbf{p}^{N}(t+\Delta t)\right]: 4,9,14$

$$
\begin{aligned}
& \mathbf{r}(t+\Delta t)=\mathbf{r}(t)+\Delta t \mathbf{v}(t)+\frac{1}{2}(\Delta t)^{2} \mathbf{a}(t) \\
& \mathbf{v}(t+\Delta t)=\mathbf{v}(t)+\frac{1}{2}(\Delta t)[\mathbf{a}(t)+\mathbf{a}(t+\Delta t)]
\end{aligned}
$$

onde $\boldsymbol{a}(t)$ é a aceleração dada pela força resultante experimentada por uma partícula. O intervalo de tempo $\Delta t$ tipicamente utilizado em simulação MD é da ordem de 1.0-10.0 fs.

Condições de contorno também são necessárias para a propagação da trajetória das partículas em simulação MD. Condições periódicas de contorno resolvem dois problemas em simulações MD: a inviabilidade computacional de se trabalhar com número muito elevado de partículas e os efeitos de superfície. Pela condição periódica de contorno ilustrada na Figura 3, as partículas contidas na caixa de simulação possuem imagens em caixas réplicas em todas as direções, de modo que, caso uma partícula ultrapasse uma das faces, sua imagem entra pela face oposta, conservando assim o número total de partículas na caixa, caso o ensemble considerado seja de $N$ constante. Finalmente, o ciclo de atualização de $\left[\mathbf{r}^{N}(t), \mathbf{p}^{N}(t)\right]$ indicado pelas Equações 12 e 13 é repetido muitas vezes até tempo total de simulação da ordem de nanossegundos.

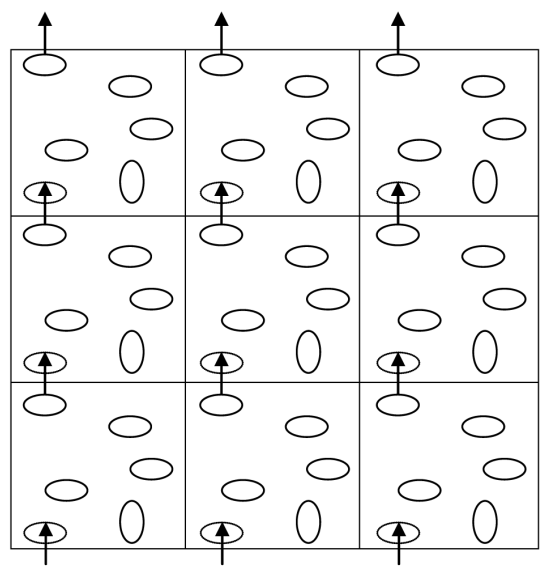

Figura 3. Representação da condição periódica de contorno utilizada em simulações $M D$

\section{Propriedades dinâmicas de fluidos}

\section{Sob o ponto de vista macroscópico}

Considere um escoamento laminar, paralelo e uniforme como na Figura 4, onde a camada inferior permanece em repouso e uma força tangencial $\mathbf{F}$ traciona a camada superior deslocando-a da direita para a esquerda com velocidade $u$. A força $\mathbf{F}$ gera uma tensão de cisalhamento $\tau$ entre a camada superior e o fluido adjacente a ela, de tal modo que um elemento de fluido inicialmente em repouso é deformado. Assumindo que a relação entre a tensão de cisalhamento $\tau$ e a taxa de deformação $\Delta u / \Delta z$ das lâminas de fluido é linear, no limite obtemos: ${ }^{3,5}$

$\tau=\eta \frac{d u}{d z}$ 
onde a constante de proporcionalidade $\eta$ é a viscosidade definida por Newton.

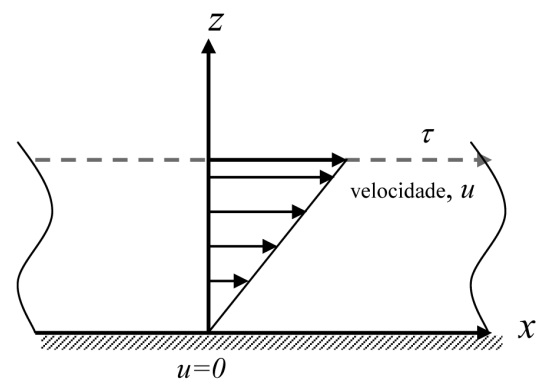

Figura 4. Modelo de um escoamento laminar, paralelo e uniforme, onde $\tau$ é a tensão de cisalhamento entre a camada superior e o fluido adjacente, e u a componente $x$ da velocidade do fluido

A viscosidade do fluido descreve a resistência ao fluxo e, microscopicamente, está relacionada às forças intermoleculares. Simulações CFD precisam do conhecimento prévio da viscosidade, ou seja, a viscosidade é um dado de entrada necessário para se estudar o comportamento do fluxo de um fluido sob diferentes condições de temperatura e pressão. Por exemplo, a viscosidade diminui acentuadamente com aumento de temperatura, geralmente seguindo dependência tipo Arrhenius, $n=e^{E / R T}$, onde $E_{a}$ é a energia de ativação. ${ }^{15} \mathrm{~A}$ taxa de condução de calor, ou fluxo de energia associado ao movimento térmico, $q$, é proporcional ao gradiente de temperatura. Considerando diferença de temperatura entre as camadas na Figura 4, a lei de Fourier é: ${ }^{3,5,10-13,16}$

$\dot{q}_{z}=-\kappa \frac{\partial T}{\partial z}$

onde $\kappa$ é o coeficiente de condutividade térmica. Esses conhecimentos fenomenológicos são necessários para o cálculo de fluxos em diferentes condições termodinâmicas pelo modelo contínuo.

\section{Sob o ponto de vista microscópico}

O conceito fundamental em simulações MD para expressar a dinâmica do sistema molecular é a função de correlação no tempo de uma dada propriedade $A[\mathbf{p}(t), \mathbf{r}(t)]$. A função de correlação no tempo clássica, $C_{A}(t)$, é definida por: ${ }^{4,6,7}$

$$
C_{A}(t)=\langle A(t) A(0)\rangle
$$

onde $<\ldots>$ significa média no ensemble e em origens de tempo $A(0)$.

Enquanto que coeficientes de transporte são dados de entrada em simulações CFD, eles podem ser calculados em simulações MD pelas funções de correlação no tempo. A partir da função de correlação de velocidade da partícula $i, C_{\mathrm{v}}(t)=\left\langle\mathbf{v}_{i}(t) \cdot \mathbf{v}_{i}(0)\right\rangle$, obtém-se o coeficiente de difusão: ${ }^{4,6,7}$

$D=\frac{1}{3} \int_{0}^{\infty}\left\langle\mathbf{v}_{i}(t) \cdot \mathbf{v}_{i}(0)\right\rangle d t$

Sob o ponto de vista macroscópico, $D$ é a constante de proporcionalidade entre o fluxo de matéria e o gradiente de concentração (lei de Fick de difusão). A Equação 17 é um exemplo de relações gerais conhecidas como equações de Green-Kubo, as quais relacionam um dado coeficiente de transporte com uma função de correlação no tempo correspondente. Outro exemplo é o cálculo da viscosidade: ${ }^{4,6,7,17}$
$\eta=\frac{1}{V k_{B} T} \int_{0}^{\infty}\left\langle\sigma_{x y}(t) \sigma_{x y}(o)\right\rangle d t$

onde $\sigma_{x y}$ é um componente fora da diagonal do tensor de stress, o qual depende das forças intermoleculares

As equações de Green-Kubo permitem o uso de simulação MD em condição de equilíbrio para calcular coeficientes de transporte. Procedimento similar ao experimental é realizado por métodos de simulação MD fora do equilíbrio, NEMD, onde uma perturbação externa é aplicada e a resposta do sistema é mensurada. ${ }^{4,16}$ Gradiente de temperatura pode ser aplicado a fim de se calcular a condutividade térmica do material, ou um gradiente de campo elétrico a fim de se calcular a condutividade iônica. $\mathrm{O}$ cálculo da viscosidade por simulação NEMD pode ser realizado acrescentando-se componente de velocidade ao longo do eixo $x$ em todas as partículas que estejam contidas em uma camada na extremidade da caixa de simulação ao longo do eixo $z$. Com a evolução da simulação, ocorre transferência de momento para as moléculas no seio do líquido e um regime de fluxo laminar é estabelecido na condição de estado estacionário (fluxo de Couette). A viscosidade é dada pelo quociente entre o gradiente do componente de velocidade $\mathrm{v}_{x}$ ao longo da direção $z$ e a magnitude da tensão de cisalhamento aplicada na direção $x$ na extremidade da caixa de simulação. Em outras palavras, esta estratégia NEMD implementa a Equação 14 para o cálculo da viscosidade.

Os dois métodos, MD e NEMD, devem resultar, evidentemente, no mesmo valor do coeficiente de transporte calculado. A Figura 5 ilustra os procedimentos de equilíbrio e não equilíbrio para o cálculo da viscosidade de um mesmo sistema, a saber, água líquida em condição ambiente (1000 moléculas $\mathrm{H}_{2} \mathrm{O}$ simuladas com o chamado modelo SPC/E). ${ }^{19} \mathrm{O}$ detalhe da figura da esquerda mostra a convergência da integral da Equação 18, a partir da qual obtemos $\eta$. Pelo procedimento de simulação NEMD, ${ }^{18}$ a viscosidade é obtida pela inclinação do gráfico do componente $\mathrm{v}_{x}$ em função da altura $z$, supondo que a tensão de cisalhamento $\tau$ foi aplicada ao longo da direção $x$. O mesmo valor de viscosidade foi obtido pelas simulações MD e NEMD, $\eta=1,2 \mathrm{cP}$. A linha contínua na figura da direita é o perfil de velocidade de acordo com a teoria hidrodinâmica, ou seja, se o fluido fosse representado como um contínuo, um perfil linear resultaria para o fluxo laminar de Couette.

\section{Simulação híbrida atomístico-contínuo}

Simulações MD reproduzem, com resolução atomística, propriedades macroscópicas necessárias para a solução das equações de Navier-Stokes e que são dados de entrada em simulações CFD. ${ }^{16,18,20-22}$ Adicionalmente, simulações atomísticas podem ser utilizadas para se analisar as próprias suposições assumidas em métodos CFD. Por exemplo, a condição de aderência é verificada por simulação NEMD como dependente da magnitude das forças de interação entre as partículas do fluido e da parede. ${ }^{23,24}$ Comportamento não Newtoniano, ou seja, viscosidade dependente da magnitude da deformação, é observado por simulações NEMD mesmo em líquidos simples. ${ }^{25-27} \mathrm{O}$ comportamento usual de dinâmica de fluidos em escala macroscópica tem sido revisto por simulações NEMD quando se trata da dinâmica de fluidos nas dimensões reduzidas, próprias da nanotecnologia..$^{22,28}$ Nesta linha, propriedades estruturais e dinâmicas de nanofluidos ${ }^{29} \mathrm{e}$ nanorreologia de alcanos líquidos têm sido calculadas ${ }^{30}$ por simulações NEMD. Em escala nanométrica, simulações NEMD revelaram a falha da lei de Fourier, ${ }^{22}$ a qual é uma relação assumida a priori em CFD. Simulações NEMD de polímeros em solução revelam deformação e alongamento da cadeia em situações de fluxo, a dependência da viscosidade com a taxa de deformação, as diferentes 

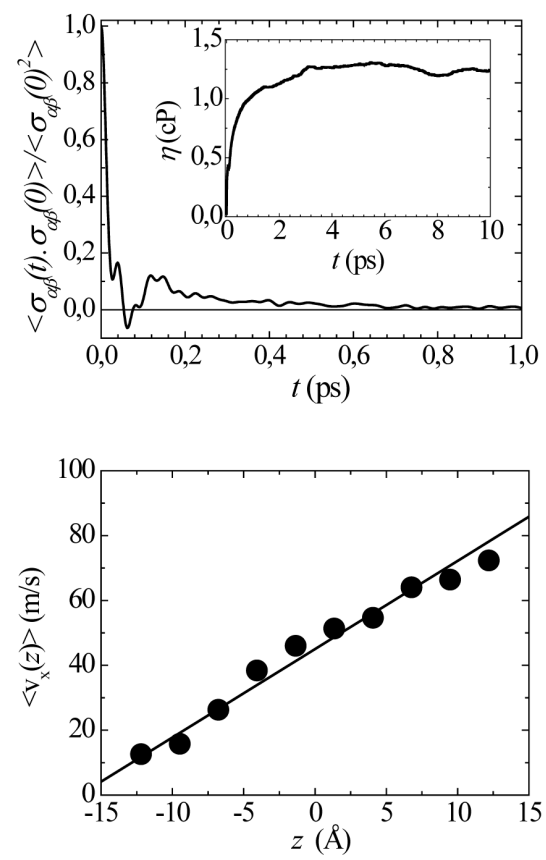

Figura 5. Cálculo da viscosidade de $\mathrm{H}_{2} \mathrm{O}$ em condição ambiente por simulação $M D$ de equilíbrio (esquerda) e de não equilíbrio (direita), utilizando o modelo $S P C / E .^{46}$ A figura da esquerda mostra a função de correlação normalizada dos elementos fora da diagonal do tensor de stress e, no detalhe, a integral da mesma em função do limite de integração, da qual se obtém a viscosidade, Equação 18. A figura da direita mostra o gradiente de $v_{x}$ ao longo da direção z, uma vez alcançado o estado estacionário devido a uma força de cisalhamento aplicada ao longo de x nas extremidades da caixa de simulação. A linha contínua é o perfil de velocidade linear previsto por hidrodinâmica

escalas dinâmicas de processos de relaxação etc. ${ }^{31-33}$ Nesses casos, o esforço computacional pode estar mais localizado no tratamento de um número elevado de moléculas de solvente do que no próprio modelo do polímero, que é o interesse central do estudo. Portanto, o desenvolvimento de metodologias híbridas NEMD/CFD é particularmente interessante, uma vez que o sistema pode ser aumentado tratando parte do mesmo como um meio contínuo.

Apesar do sucesso alcançado pelas simulações MD, há uma lacuna do ponto de vista macroscópico somente com o estudo atomístico devido a limitações de tamanho do sistema e tempo de simulação. Hadjiconstantinou ${ }^{34}$ considerou o fluxo de dois fluidos imiscíveis em um canal bidimensional, cuja parede foi descrita com um modelo de esferas rígidas. A dinâmica na proximidade do ponto de contato entre os fluidos e a parede foi estudada com um modelo híbrido, sendo obtidos resultados melhores que estudos anteriores de simulações puramente atomística.

Originalmente, métodos híbridos foram desenvolvidos com o objetivo de se estudar o fluxo de gases, porque são de mais fácil implementação que fluidos densos..$^{35}$ Métodos híbridos NEMD/CFD foram utilizados no estudo da deformação de uma cadeia polimérica em solução em função da tensão de cisalhamento ${ }^{36}$ e no estudo da linha de contato entre dois líquidos imiscíveis sob fluxo. ${ }^{34}$ Recentemente, simulações híbridas atomístico-contínuo têm sido empregadas em uma gama de problemas no estudo da matéria condensada, desde modelos de esferas rígidas, ${ }^{34}$ fluidos Lennard-Jones, ${ }^{37,38}$ líquidos moleculares ${ }^{39}$ e polímeros fundidos. ${ }^{36} \mathrm{~A}$ investigação da mecânica de fluidos em nanoescala é motivada pelo desenvolvimento de nanodispositivos, tais como biossensores. Werder et al. ${ }^{40}$ investigaram o fluxo de argônio em torno de um nanotubo de carbono, onde a descrição atomística era restrita ao nanotubo de carbono e átomos de argônio vizinhos.
Fabritis et al.$^{41}$ utilizaram simulações híbridas para estudar a interface de uma monocamada lípidica solvatada por moléculas de água, sendo que o solvente foi tratado em diferentes subdomínios, partindo de uma descrição molecular próximo da camada lípidica, passando por subdomínio híbrido atomístico-contínuo, até um tratamento contínuo pelas equações da hidrodinâmica.

Nos métodos híbridos, uma parte do sistema é discreta e tratada por NEMD, outra parte é contínua e tratada por CFD, e há uma região da caixa de simulação onde há uma sobreposição dos subdomínios, como mostra a Figura 6A. Na região de sobreposição ocorre a troca de informação entre os procedimentos NEMD e CFD: médias são computadas na parte NEMD, servindo como condição de contorno para CFD, enquanto que os campos da CFD são usados para corrigir e adequar os valores das variáveis NEMD. O ganho de eficiência do método híbrido depende da dimensão relativa entre os diferentes subdomínios e das implementações numéricas dos modelos contínuo e atomístico. Uma formulação mais simples consiste de dois subdomínios distintos separados por uma interface (Figura 6B), onde o aspecto importante é a continuidade das propriedades termodinâmicas e de transporte através da interface entre os dois subdomínios.

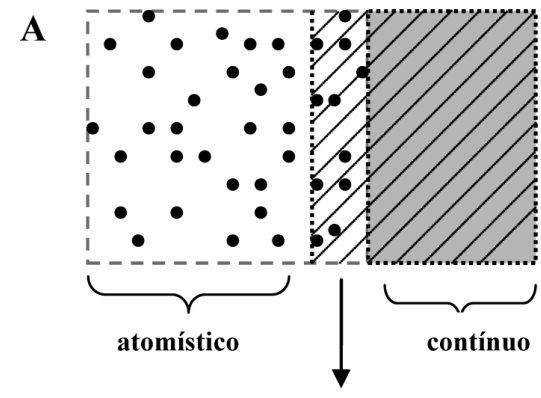

sobreposição

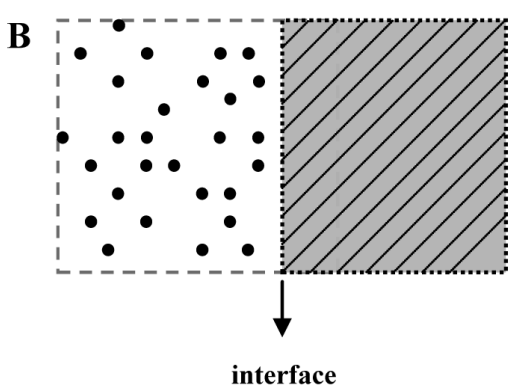

Figura 6. Modelos híbridos contínuo-atomístico contendo uma região de sobreposição (A) ou uma interface simples $(B)$

Dois esquemas gerais de acoplamento das regiões contínua e atomística podem ser distinguidos: troca de fluxo direto ${ }^{42-44}$ (direct flux exchange) e método de decomposição de domínio (Schwartz coupling method) ${ }^{37} \mathrm{O}$ primeiro é baseado no trabalho original de O'Connel e Thompson, ${ }^{45}$ onde ocorre fluxo de massa, momento e energia diretamente através da interface (Figura 6B). O método de Schwartz é baseado no acoplamento entre os domínios por intermédio de subdomínios de sobreposição, os quais são formados tanto pela descrição atomística quanto contínua (Figure 6A). O método de Schwartz é indicado tanto para fluidos compressíveis e incompressíveis, exigindo desacoplamento adequado das escalas de tempo e distância das soluções nos diferentes subdomínios. Esta questão surge pelo fato do tempo de integração do subdomínio contínuo ser diferente do tempo de integração do subdomínio molecular. Em geral, para cada 
100 passos de $\mathrm{MD}$, calcula-se a média das propriedades que serão necessárias para uma integração numérica das equações de NavierStokes no subdomínio contínuo, repetindo o ciclo de integração até que o estado estacionário seja atingido.

Utilizaremos água líquida para ilustrar uma simulação híbrida do fluxo de Couette, a qual, sob o ponto de vista de CFD, é a resolução numérica da equação diferencial:

$\frac{\partial \mathrm{v}_{x}}{\partial t}=\eta \frac{\partial^{2} \mathrm{v}_{x}}{\partial z^{2}}$

A equação acima foi resolvida para o campo de velocidade com um algoritmo de Euler explícito, ${ }^{5}$ isto é, Equação 9, onde a discretização espacial foi aproximada pela diferença finita:

$\left.\frac{\partial^{2} \mathbf{v}_{x}}{\partial z^{2}}\right|_{i} \approx \frac{\mathrm{v}_{x, i+1}^{t}-2 \mathbf{v}_{x, i}^{t}+\mathrm{v}_{x, i-1}^{t}}{(\Delta z)^{2}}$

Em CFD, a viscosidade $\eta$ é um dado de entrada obtido experimentalmente mas, na versão híbrida, pode ser o valor determinado previamente por simulação MD do sistema em questão.

No procedimento híbrido, consideramos a camada superior e inferior ao longo do eixo $\mathrm{z}$ da caixa de simulação NEMD como regiões de sobreposição entre a descrição atomística e contínua. Portanto, o sistema inteiro é composto de uma parte discreta na região $-11<$ $z<11 \AA$, entre duas partes contínuas que estendem o tamanho total do sistema até $z= \pm 30 \AA$, com regiões de sobreposição definidas por paredes de espessura $4 \AA$ (Figura 7). Condições de contorno periódicas para a parte atomística (Figura 3) existem apenas ao longo das direções $x$ e $y$. O campo de velocidade médio nos extremos em $z$ da região atomística atua como a condição de contorno para a parte $\mathrm{CFD}$, assim como as velocidades das partículas nessa região de sobreposição são corrigidas para serem consistentes com o campo de velocidade da parte CFD.

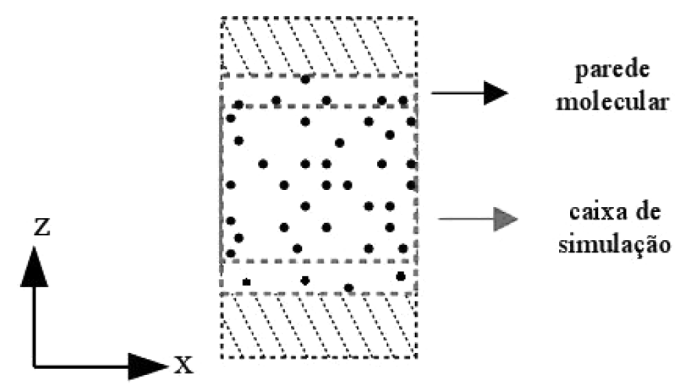

Figura 7. Modelo híbrido contínuo-atomístico utilizado em uma simulação de água contendo uma região central atomística, regiões superior e inferior contínuas e uma região de sobreposição

Simulações NEMD foram realizadas com o modelo SPC/E para $\mathrm{H}_{2} \mathrm{O},{ }^{46}$ cuja função energia potencial inclui interações de curto alcance do tipo Lenard-Jones e interações de Coulomb de longo alcance. O problema das interações de longo alcance em simulações $\mathrm{MD}^{4}$ foi tratado pelo método de Wolf. ${ }^{47}$ As simulações NEMD consideraram 1000 moléculas de $\mathrm{H}_{2} \mathrm{O}$, tempo de integração das equações de movimento $\Delta t=1,0$ fs e o ensemble $N P T$, onde o controle de pressão e temperatura foi realizado com o barostato e termostato de Berendsen. ${ }^{48} \mathrm{~A}$ fim de validar a metodologia, diferentes condições de fluxo foram testadas variando-se a tensão de cisalhamento ao longo da direção $x$ nas extremidades da caixa de simulação.

A Figura 8 ilustra o resultado da simulação híbrida. A linha contínua é o perfil linear de velocidade para um fluxo laminar de Couette, de acordo com a teoria hidrodinâmica, obtido pelo procedimento CFD, enquanto que o perfil de velocidade da parte discreta do sistema é mostrado pelos círculos pretos. O regime de estado estacionário é alcançado em cerca de $200 \mathrm{ps}$, quando a parte atomística adquire o perfil de velocidade correto de acordo com o campo de velocidade da parte CFD atuando nos extremos da caixa de simulação NEMD.

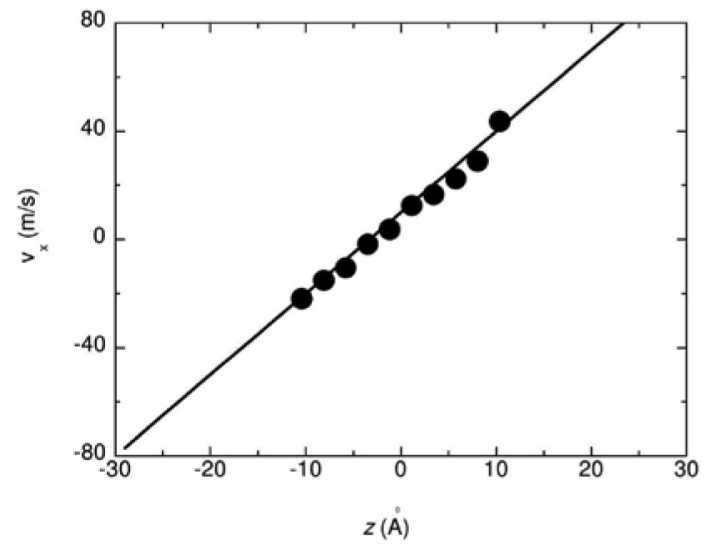

Figura 8. Perfil de velocidade obtido por uma simulação híbrida NEMD/ CFD de água. A parte central do sistema (círculos pretos) é uma simulação atomística contendo 1000 moléculas $\mathrm{H}_{2} \mathrm{O}$. A parte atomística reside entre duas partes contínuas simuladas por CFD (perfil de velocidade dado pela linha contínua) com as quais são trocadas informações do campo de velocidade

\section{CONCLUSÃO}

Uma visão microscópica do fluxo de fluidos é de grande importância para o entendimento dos processos relacionados de conservação de massa, momento e energia. Propriedades dinâmicas calculadas a partir da técnica CFD podem ser compreendidas com detalhe molecular quando usada em conjunto com técnicas atomísticas, como a simulação por dinâmica molecular de equilíbrio e não equilíbrio. Neste sentido, simulações híbridas atomístico-contínuo podem contribuir tanto na caracterização do fluxo de fluidos quanto em aplicações tecnológicas e problemas de engenharia. De fato, o uso de técnicas híbridas permite o estudo de fenômenos físico-químicos em meso/microescala, servindo de auxílio a técnicas experimentais na investigação do fluxo de fluidos no limite hidrodinâmico, bem como contribuindo na predição de propriedades dinâmicas, tais como coeficiente de difusão e viscosidade.

\section{AGRADECIMENTOS}

À FAPESP e ao CNPq pelo auxílio financeiro.

\section{REFERÊNCIAS}

1. Aristóteles; Physics, Oxford University Press: Oxford, 1996.

2. Lucrécio; Da Natureza (De Rerum Natura), Ediouro: Rio de Janeiro, 1988.

3. Fox, R. W.; McDonald, A. T.; Introdução à Mecânica dos Fluídos, Guanabara Koogan: Rio de Janeiro, 1992.

4. Allen, M. P.; Tildesley, D. J.; Computer Simulation of Liquids, Oxford Science Publications: London, 1987.

5. Fortuna, A. de O.; Técnicas Computacionais para Dinâmica dos Fluidos, EDUSP: São Paulo, 2000

6. Hansen, J. P.; McDonald, I. R.; Theory of Simple Liquids, $3^{\text {rd }}$ ed., Academic Press: London, 2006. 
7. McQuarrie, D. A.; Statistical Mechanics, University Science Books: New York, 1976.

8. Hoover, W. G.; Ann. Rev. Phys. Chem. 1983, 34, 103.

9. Frenkel, D.; Smit, B.; Understanding Molecular Simulations. From Algorithms to Applications, Academic Press: San Diego, 1996.

10. Fried, J.; Numerical simulation of viscous flow: A study of molecular dynamics and computational fluid dynamics, falta editora: Virginia, 2007.

11. Ferziger, J. H.; Peric, M.; Computational methods for fluid dynamics, $3^{\text {rd }}$ ed.; Springer Verlag: Berlin, 1999.

12. Schmidt, F. W.; Henderson, R. E.; Wolgemuth, C. H.; Introducão às Ciências Térmicas, Edgard Blücher: São Paulo, 1996.

13. Sadus, R. J.; Molecular Simulations of Fluids: Theory, Algorithms, and Object-orientation, Elsevier: London, 1999.

14. Swope, W. C.; Andersen, H. C.; Berens, P. H.; Wilson, K. R.; J. Chem. Phys. 1982, 76, 637.

15. Atkins, P. W.; de Paula, J.; Physical Chemistry, Oxford University Press: London, 2008.

16. Evans, D. J.; Morris, G. P.; Statistical Mechanics of Nonequilibrium Liquids, Academic Press: London, 1990.

17. Hess, B.; J. Chem. Phys. 2002, 116, 209.

18. Ashurst, W. T.; Hoover, W. G.; Phys. Rev. A 1975, 11, 658.

19. Berendsen, H. J. C.; Grigera, J. R.; Straatsma, T. P.; J. Phys. Chem. 1987, 91, 6269 .

20. Sarman, S. S.; Evans, D. J.; Cummings, P. T.; Phys. Rep. 1998, 305, 1.

21. Koplik, J.; Banavar, J. R.; Annu. Rev. Fluid Mech. 1995, $27,257$.

22. Todd, B. D.; Comput. Phys. Commun. 2001, 142, 14.

23. Thompson, P. A.; Robbins, M. O.; Phys. Rev. A 1990, 41, 6830.

24. Leng, Y. S.; Cummings, P. T.; J. Chem. Phys. 2006, 125, 104701.

25. Heyes, D. M.; J. Non-Newtonian Fluid Mech. 1986, 21, 137.

26. Angelani, L.; Ruocco, G.; Sciortino, F.; Tartaglia, P.; Zamponi, F.; Phys. Rev. E 2002, 66, 061505.

27. Varnik, F.; Bocquet, L.; Barrat, J.-L.; J. Chem. Phys. 2004, 120, 2788.
28. Gad-el-Hak, M.; Phys. Fluids 2005, 17, 100612.

29. Pozhar, L. A.; Phys. Rev. E 2000, 61, 1432.

30. Gupta, S. A.; Cochran, H. D.; Cummings, P. T.; Fluid. Phase. Equilibria 1998, $125,150$.

31. Pierleoni, C.; Ryckaert, J.-P.; Macromol. 1995, 28, 5097.

32. Aust, C.; Kröger, M.; Hess, S.; Macromol. 1999, 32, 5660.

33. Cifre, J. G. H.; Hess, S.; Kröger, M.; Macromol. Theory Simul. 2004, 13,748 .

34. Hadjicostantinou, N. G..; Phys. Rev. E 1999, 59, 2575.

35. Wadsworth, D. C.; Erwin, D. A.; AIAA Paper 1992, 92, 2975.

36. Barsky, S.; Delagado-Buscalioni, R.; Coveney, P. V.; J. Chem. Phys. 2004, 121, 2403.

37. Werder, T.; Walther, J. H.; Koumoutsakos, P.; J. Comput. Phys. 2005 , 205, 373.

38. Kotsalis, E. M.; Walther, J. H.; Koumoutsakos, P.; Phys. Rev. E 2007, 76, 016709

39. Delgado-Buscalioni, R.; Kremer, K.; Praprotnik, M.; J. Chem. Phys. 2008, 128,114110 .

40. Werder, T.; Walther, J. H.; Jaffe, R. L.; Halicioglu, T.; Koumoutsakos, P.; J. Phys. Chem. B 2003, 107, 1349.

41. De Fabritis, G.; Delgado-Buscalioni, R.; Coveney, P. V.; Phys. Rev. Lett. 2006, 97, 134501.

42. Hadjicostantinou, N. G.; Phys. Rev. E 1999, 59, 2575.

43. Delgado-Buscalioni, R.; Coveney, P. V.; Phys. Rev. E 2003, 67, 046704.

44. Delgado-Buscalioni, R.; Flekkoy, E. G.; Coveney, P. V.; Europhys. Lett. 2005, 69, 959 .

45. O'Connell, S. T.; Thompson, P. A.; Phys.Rev. E 1995, 52, R5792.

46. Berendsen, H. J. C.; Grigera, J. R.; Straatsma, T. P.; J. Phys. Chem. 1987, 91, 6269.

47. Wolf, D.; Keblinski, P.; Phillpot, S. R.; Eggebrecht, J.; J. Chem. Phys. 1999, $110,8254$.

48. Berendsen, H. J. C.; Postma, J. P. M.; Gunsteren, W. F.; DiNola, A.; Haak, J. R.; J. Chem. Phys. 1984, 81, 3684. 